\title{
Desenvolvimento ponderal e parâmetros hematológicos e bioquímicos de cordeiros Pantaneiros submetidos a diferentes manejos de amamentação
}

\author{
[Ponderal development and hematological and biochemical parameters of Pantaneiro lambs \\ subjected to different suckling regimens] \\ P.H. Braz ${ }^{1}$, M.M. Castro ${ }^{2}$, K. Cansian², A.I. Souza ${ }^{2}$, F.M. Vargas Júnior ${ }^{3}$, L.G. Ávila ${ }^{2}$ \\ ${ }^{1}$ Instituto Federal de Educação, Ciência e Tecnologia Farroupilha - Frederico Westphalen, RS \\ ${ }^{2}$ Universidade Federal de Mato Grosso do Sul - Campo Grande, MS \\ ${ }^{3}$ Universidade Federal da Grande Dourados - Dourados, MS
}

\begin{abstract}
RESUMO
Este estudo teve por objetivo avaliar o desenvolvimento ponderal e a dinâmica dos parâmetros hematológicos e bioquímicos de cordeiros Pantaneiros submetidos a diferentes manejos de amamentação dos 15 aos 43 dias. Foram separados 30 cordeiros em três diferentes grupos $(n=10)$. Os grupos foram caracterizados conforme o tempo de permanência das ovelhas com suas crias em diferentes sistemas de amamentação: MAM24 - ovelhas e cordeiros 24 horas em conjunto; MAM12 - ovelhas e cordeiros 12 horas em conjunto durante a noite; MAM2x30 - ovelhas e cordeiros 30 minutos de manhã e 30 minutos à tarde em conjunto. As coletas de amostras sanguíneas e as pesagens ocorriam a cada sete dias. Houve aumento significativo no peso com o avanço da idade dos cordeiros nos três manejos, mas os tratamentos não diferiram entre si para o ganho de peso. Houve diferenças entre os três tratamentos para a variável hematológica CHGM e para as variáveis bioquímicas AST, glicose, ureia e proteína total. As variáveis bioquímicas foram influenciadas pela faixa etária dos animais. Os diferentes manejos de amamentação não influenciaram o desenvolvimento ponderal dos cordeiros. Não ocorreram alterações patológicas. Alguns parâmetros hematológicos e bioquímicos podem ser influenciados pelo desenvolvimento etário dos cordeiros.
\end{abstract}

Palavras-chave: crescimento, glicemia, ovelhas, ureia

\begin{abstract}
This study aimed to evaluate the weight development and the dynamics of hematological and biochemical parameters of Pantaneiro lambs submitted to different breastfeeding management from 15 to 43 days. First, 30 lambs were separated into three different groups $(n=10)$. The groups were characterized according to the length of stay of ewes with their young in different breastfeeding systems: MAM24 - ewes and lambs 24 hours together. MAM12- Sheep and lambs 12 hours together at night. MAM2x30 - sheep and lambs 30 minutes in the morning and 30 minutes in the afternoon together. Blood samples were collected and weighed every seven days. Significant increase in weight was observed with the age of the lambs in the three managements, but the treatments did not differ for weight gain. Differences were identified between the three treatments for the hematological variable CHGM and for the biochemical variables AST, glucose, urea and total protein. Correlating the age of the lambs with the biochemical variables, there was variation influenced by the age of the animals. The different management of breastfeeding did not influence the weight development of the lambs. Hematological and biochemical variations did not represent pathological changes. Some hematological and biochemical parameters may be influenced by the age development of lambs.
\end{abstract}

Keywords: growth, blood glucose, sheep, urea

Recebido em 7 de fevereiro de 2020

Aceito em 6 de abril de 2020

E-mail: paulo.braz@iffarroupilha.edu.br 


\section{INTRODUÇÃO}

Os ovinos Pantaneiros representam um grupamento genético cujos animais possuem elevada rusticidade, boa habilidade materna e importantes características reprodutivas. Esses animais passaram por seleção genética natural em decorrência das condições climáticas características da região do Pantanal (Mora et al., 2015). O que se conhece sobre os ovinos Pantaneiros está relacionado à caracterização morfoestrutural (Oliveira et al., 2014), às características da carne e da carcaça (Vargas Junior et al., 2015) e à produção e qualidade do leite (Longo et al., 2018).

A amamentação controlada é considerada uma alternativa para aumentar a eficiência reprodutiva e produtiva dos ovinos, uma vez que proporciona às ovelhas o retorno ao estro pós-parto mais precoce. Esse retorno à fase estral beneficia a antecipação de uma nova concepção, o que reduz o intervalo entre partos e, consequentemente, aumenta a produção animal (Leal et al., 2010). Avaliar os cordeiros em relação ao seu ganho de peso torna-se importante nesse contexto de amamentação restrita, principalmente para verificar possíveis alterações relacionadas ao crescimento dos cordeiros. Os exames laboratoriais são frequentemente obtidos para auxiliar a avaliação do estado sanitário de um animal individualmente ou de um determinado rebanho, e também para identificar condições patológicas e confirmar diagnósticos (Šoch et al., 2011).

Diante do contexto, objetivou-se com o estudo avaliar o desenvolvimento ponderal e o comportamento de alguns componentes sanguíneos de cordeiros do grupamento genético Pantaneiro em três diferentes manejos de amamentação.

\section{MATERIAL E MÉTODOS}

O experimento foi desenvolvido no Centro de Pesquisa de Ovinos da Faculdade de Ciências Agrárias da Universidade Federal da Grande Dourados (UFGD), no município de Dourados/MS.Todos os procedimentos experimentais foram realizados mediante autorização do Comitê de Ética no Uso de Animais (Ceua-UFGD, sob o protocolo número 17/2016). Os animais selecionados eram pertencentes ao grupamento genético Pantaneiro. Foram utilizadas 60 ovelhas Pantaneiras, entre nulíparas e pluríparas, submetidas ao processo de sincronização de estro, adotando-se o uso de esponjas contendo progesterona, as quais permaneceram nas fêmeas por cinco dias.

Após seis dias de inserção das esponjas, as fêmeas receberam uma dose de prostaglandina e, no dia subsequente, foram expostas aos reprodutores. A intenção da sincronização de estro foi a obtenção de lotes homogêneos de cordeiros. Após o nascimento, foram selecionados 30 cordeiros, os quais permaneceram com as mães até os 15 dias de vida. Após esse período, os animais foram divididos em três grupos de 10 animais.

A distribuição dos animais em cada grupo referente ao manejo de amamentação ocorreu inteiramente ao acaso e o experimento teve início quando os cordeiros atingiram 15 dias de idade, estendendo-se até o $43^{\circ}$ dia. No período compreendido entre o nascimento e os primeiros 15 dias de vida, os cordeiros permaneceram com suas mães em tempo integral em sistema semiintensivo, sendo permitido o acesso à pastagem (Brachiaria brizantha cv. Piatã) e ao creep feeding com ração comercial $(120 \mathrm{~g} / \mathrm{kg}$ de proteína bruta) durante o dia; no período da noite, os animais eram mantidos em aprisco coberto. No $15^{\circ}$ dia de vida, as fêmeas e seus respectivos cordeiros foram distribuídos aleatoriamente em grupos submetidos a diferentes tipos de manejo de amamentação, conforme descrito abaixo:

MAM24 - ovelhas e cordeiros permaneciam 24 horas juntos; MAM12 - ovelhas e cordeiros permaneciam 12 horas juntos durante o período da noite; MAM2X30 - ovelhas e cordeiros permaneciam 30 minutos juntos de manhã e novamente 30 minutos à tarde.

Os cordeiros pertencentes aos três grupos de manejo de amamentação foram colocados sob o mesmo sistema alimentar (Brachiaria brizantha cv. Piatã), ração comercial no creep feeding $(120 \mathrm{~g} / \mathrm{kg}$ de proteína bruta) e água ad libitum. Durante o período experimental (de 15 a 43 dias de vida), a cada sete dias os cordeiros foram submetidos à pesagem. Realizava-se também avaliação física para verificar possíveis alterações da condição clínica em decorrência de enfermidades (inspeção, palpação da região umbilical e avaliação de mucosas aparentes) e 
eram realizadas coletas de sangue por venopunção jugular.

As amostras sanguíneas eram coletadas com o volume mínimo de $4 \mathrm{~mL}$ de sangue venoso, utilizando-se agulhas descartáveis de coleta a vácuo, sendo o sangue acondicionado em tubo com anticoagulante do tipo EDTA para análises hematológicas e glicêmicas. Para a determinação dos parâmetros bioquímicos (concentrações séricas de creatinina, ureia, proteínas totais e albumina, e atividade da enzima aspartato aminotransferase (AST), as amostras foram acondicionadas em tubos sem anticoagulante.

As amostras destinadas à mensuração da glicemia foram coletadas e imediatamente processadas. Para avaliação dos parâmetros hematológicos, as amostras foram resfriadas a $4^{\circ} \mathrm{C}$ até o momento da análise, que não excedeu o período de seis horas após o momento da coleta. Para determinação dos parâmetros bioquímicos, as amostras foram mantidas em repouso até a formação do coágulo sanguíneo e posteriormente centrifugadas a 4000rpm, durante cinco minutos. O soro obtido foi armazenado a $-20^{\circ} \mathrm{C}$ até o processamento das análises.

A contagem total de eritrócitos, o volume globular, a concentração plasmática de hemoglobina, o volume corpuscular médio (VGM), a concentração de hemoglobina corpuscular média (CHGM) e a contagem total de leucócitos foram determinados com o auxílio de analisador hematológico (pocH-100 iVDiff ${ }^{\circledR}$ ). As análises bioquímicas das concentrações séricas de creatinina, ureia, proteínas totais e albumina e da atividade sérica da enzima AST foram realizadas com o auxílio de analisador bioquímico automático (cobas c $111^{\circledR}$ ) e a mensuração da glicemia foi realizada mediante utilização de equipamento portátil próprio para mensuração da concentração plasmática de glicose (Accu-Chek Active $^{\circledR}$ ).

$\mathrm{O}$ experimento foi conduzido com delineamento inteiramente ao acaso. A normalidade dos resultados de cada tratamento em cada tempo foi avaliada pelo teste de Shapiro-Wilk, e a homogeneidade de variâncias foi verificada pelo teste de Bartlett. Para a identificação de efeito dos tratamentos nas diferentes idades, as variáveis de desempenho (peso e ganho de peso no período experimental) foram submetidas à análise de variância. As variáveis bioquímicas (concentrações séricas de creatinina, ureia, proteínas totais, albumina e globulinas, atividade sérica da enzima AST e glicemia) e hematológicas (contagem total de leucócitos, quantidade total de eritrócitos, concentração plasmática de hemoglobina, volume globular, VGM e CHGM) foram submetidas ao teste não paramétrico de Kruskal-Wallis seguido do teste bilateral não paramétrico de Dunn.

Os coeficientes angulares dos modelos de regressão linear do peso em função da idade foram testados pelo teste t de Student, e o teste F foi empregado na avaliação da identidade dos modelos. Nas variáveis bioquímicas e hematológicas, o efeito do tempo nos diferentes tratamentos foi avaliado pelo teste de Friedman, seguido do teste Wilcoxon com a correção de Bonferroni. O nível de significância adotado em toda a análise estatística foi $\mathrm{P}=0,05$, com auxílio do programa estatístico Bioestat (Zar, 2010).

\section{RESULTADOS}

No período compreendido entre 15 e 43 dias de vida, os três diferentes tipos de manejo de amamentação não influenciaram $o$ desenvolvimento dos cordeiros, embora tenha sido observado aumento no peso com o avanço da idade dos cordeiros nos três tratamentos $(\mathrm{P}<0,05)$. Os parâmetros das três regressões não diferiram entre si $(\mathrm{P}>0,05)$ e estimaram o mesmo modelo linear. Os valores estabelecidos para as variáveis hematológicas dos cordeiros entre 15 e 43 dias de idade encontram-se listados na Tab. 1, sendo comparados aos intervalos de referência relatados para ovinos, compilados por Byers e Kramer (2010). 
Tabela 1. Valores de mediana* (Md) e distância interquartílica (DQ) das variáveis hematológicas contagem total de eritrócitos, volume globular, concentração plasmática de hemoglobina, volume corpuscular médio (VGM), concentração de hemoglobina corpuscular média (CHGM) e contagem total de leucócitos, obtidas de sangue venoso coletado de cordeiros Pantaneiros submetidos a diferentes manejos de amamentação do $15^{\circ}$ ao $43^{\circ}$ dia de vida

\begin{tabular}{|c|c|c|c|c|c|c|c|c|}
\hline \multirow[t]{2}{*}{ Idade } & \multirow[t]{2}{*}{ Variáveis hematológicas } & \multicolumn{2}{|c|}{ MAM24** } & \multicolumn{2}{|c|}{ MAM12** } & \multicolumn{2}{|c|}{ MAM2X30** } & \multirow[t]{2}{*}{ Valor-P } \\
\hline & & $\mathrm{Md}$ & DQ & Md & DQ & $\mathrm{Md}$ & DQ & \\
\hline \multirow{6}{*}{15 dias } & Hemácias $\left(\times 10^{6}\right)$ & 10,27 & 1,30 & 11,26 & 0,88 & 11,13 & 0,72 & 0,282 \\
\hline & Volume globular (\%) & 32,40 & 2,30 & 34,15 & 1,65 & 36,45 & 6,60 & 0,227 \\
\hline & Hemoglobina (g/dL) & 8,95 & 0,95 & 9,51 & 0,35 & 9,45 & 1,48 & 0,127 \\
\hline & VGM (fL) & 31,45 & 3,20 & 30,85 & 1,48 & 30,95 & 2,63 & 0,934 \\
\hline & CHGM (\%) & 27,75 & 1,40 & 27,85 & 0,65 & 27,25 & 0,95 & 0,563 \\
\hline & Leucócitos totais $\left(\times 10^{3}\right)$ & 6,45 & 1,90 & 7,30 & 2,03 & 7,90 & 1,60 & 0,196 \\
\hline \multirow{5}{*}{22 dias } & Hemácias $\left(x 10^{6}\right)$ & 11,79 & 1,20 & 11,04 & 0,85 & 12,01 & 1,90 & 0,112 \\
\hline & Volume globular (\%) & 35,10 & 4,10 & 33,10 & 1,40 & 36,35 & 2,85 & 0,990 \\
\hline & Hemoglobina (g/dL) & 9,10 & 1,05 & 9,30 & 0,42 & 9,65 & 0,88 & 0,129 \\
\hline & VGM (fL) & 29,40 & 2,85 & 30,75 & 1,45 & 29,75 & 2,38 & 0,661 \\
\hline & CHGM (\%) & $26,30^{\mathrm{a}}$ & 1,43 & $27,55^{\mathrm{ab}}$ & 1,10 & $27,05^{\mathrm{b}}$ & 0,90 & 0,017 \\
\hline \multirow{7}{*}{29 dias } & Leucócitos totais $\left(\mathrm{x} 10^{3}\right)$ & 6,40 & 2,13 & 6,95 & 1,93 & 7,00 & 2,70 & 0,990 \\
\hline & Hemácias $\left(\times 10^{6}\right)$ & 12,09 & 0,47 & 11,62 & 1,62 & 11,60 & 0,94 & 0,718 \\
\hline & Volume globular (\%) & 34,85 & 3,50 & 35,85 & 5,15 & 34,95 & 2,68 & 0,971 \\
\hline & Hemoglobina (g/dL) & 9,45 & 0,88 & 9,45 & 0,75 & 9,15 & 0,28 & 0,415 \\
\hline & VGM (fL) & 29,55 & 2,05 & 28,90 & 2,28 & 29,75 & 1,95 & 0,980 \\
\hline & CHGM (\%) & 26,30 & 0,65 & 26,70 & 1,88 & 26,80 & 0,55 & 0,823 \\
\hline & Leucócitos totais $\left(\mathrm{x} 10^{3}\right)$ & 8,70 & 1,15 & 7,15 & 2,75 & 7,25 & 0,43 & 0,057 \\
\hline \multirow{5}{*}{36 dias } & Hemácias (x106) & 12,69 & 1,46 & 12,79 & 1,06 & 11,98 & 1,55 & 0,768 \\
\hline & Volume globular (\%) & 35,35 & 5,53 & 37,00 & 3,22 & 35,55 & 3,83 & 0,823 \\
\hline & Hemoglobina (g/dL) & 9,40 & 1,10 & 9,85 & 0,65 & 9,35 & 1,05 & 0,893 \\
\hline & VGM (fL) & 29,40 & 2,20 & 28,55 & 1,68 & 28,00 & 3,13 & 0,772 \\
\hline & CHGM (\%) & 25,85 & 1,25 & 26,35 & 0,97 & 25,95 & 0,95 & 0,417 \\
\hline \multirow{7}{*}{43 dias } & Leucócitos totais $\left(\times 10^{3}\right)$ & 7,95 & 3,38 & 7,90 & 1,98 & 6,90 & 2,25 & 0,351 \\
\hline & Hemácias $\left(\times 10^{6}\right)$ & 12,88 & 1,71 & 12,10 & 0,86 & 12,91 & 1,70 & 0,303 \\
\hline & Volume globular (\%) & 38,75 & 5,50 & 34,55 & 3,83 & 35,85 & 6,50 & 0,250 \\
\hline & Hemoglobina (g/dL) & 9,90 & 1,05 & 9,40 & 0,92 & 9,60 & 1,00 & 0,506 \\
\hline & VGM (fL) & 28,85 & 3,58 & 27,60 & 2,60 & 27,50 & 3,40 & 0,824 \\
\hline & CHGM (\%) & 25,75 & 1,45 & 27,10 & 1,48 & 26,75 & 1,45 & 0,133 \\
\hline & Leucócitos totais $\left(\times 10^{3}\right)$ & 8,00 & 1,83 & 7,00 & 2,85 & 7,80 & 2,63 & 0,165 \\
\hline
\end{tabular}

*Valores de mediana seguidos de letras diferentes para cada variável e idade indicam diferenças $(\mathrm{P}<0,05)$ entre os tratamentos pelo método não paramétrico de Dunn na comparação dos postos médios.**MAM24: tratamento 3cordeiros e ovelhas 24 horas juntos. **MAM12: tratamento 1 - cordeiros e ovelhas 12 horas juntos. **MAM2x30: tratamento 2- cordeiros e ovelhas juntos por 30 minutos de manhã e novamente por 30 minutos à tarde.

Aos 22 dias de vida, foi possível notar que o tratamento MAM24 e o tratamento MAM12 diferiram entre si $(\mathrm{P}<0,05)$ em relação à concentração de hemoglobina corpuscular média (CHGM). Como não foram constatados valores de volume globular, contagem total de hemácias e concentração plasmática de hemoglobina que caracterizassem quadros de anemia, a variável CHGM tem pouco valor diagnóstico para esse contexto. Não foram identificadas diferenças importantes entre os três manejos quanto aos componentes do eritrograma e do leucograma.
Semelhantemente, também não foram notadas alterações clínicas e hematológicas que justificassem quadros patológicos.

Quando foi avaliado o comportamento das células sanguíneas em relação ao tempo de vida dos cordeiros, houve aumento da contagem total de hemácias a partir dos 22 dias de vida dos cordeiros do grupo MAM24. Para os cordeiros do grupo MAM12, a contagem de hemácias apresentou aumento a partir do $29^{\circ}$ dia. Diferentemente, para os animais do tratamento de duas mamadas de 30 
minutos, houve aumento da contagem de hemácias aos 22 dias, com decréscimo aos 29 dias de vida e posterior elevação novamente aos 36 dias.

A variável volume globular médio (VGM) apresentou valores menores de acordo com o desenvolvimento etário dos animais, para todos os tratamentos. No tratamento de amamentação de 12 horas, a redução começou aos 29 dias, enquanto para os demais tratamentos o valor do VGM decresceu a partir dos 22 dias. A concentração de hemoglobina corpuscular média (CHGM) também apresentou redução de acordo com a evolução da idade dos cordeiros; para o grupo de animais que permaneceram 24 horas com as mães, os menores valores foram observados aos 36 e 43 dias de vida. Para o tratamento de 12 horas, os menores valores foram encontrados aos 36 dias. De forma semelhante, isso foi observado no grupo de cordeiros que receberam amamentação duas vezes por dia, durante 30 minutos.

A concentração plasmática de hemoglobina variou em relação ao tempo apenas no grupo de animais que permaneceram 24 horas com as mães e apresentou valores mais elevados a partir dos 22 dias. Para a variável volume globular, houve variação apenas no grupo submetido ao manejo de 24 horas; o volume globular apresentou-se mais elevado a partir dos 22 dias de vida dos cordeiros, sendo o maior valor observado aos 43 dias.

No grupo MAM12, os valores referentes à atividade enzimática da aspartato aminotransferase (AST) aumentaram aos 22 e 43 dias de vida, diferindo do grupo de cordeiros que ficavam integralmente com as ovelhas. Aos 15 dias de vida, os valores de glicemia foram maiores entre os animais dos manejos MAM2x30 e MAM24, quando comparados ao tratamento MAM12. Com 22 dias, foi possível notar diferença apenas entre os grupos de animais que permaneciam 12 horas e 24 horas com as mães. Com a evolução da idade, não se observou diferença para esta variável entre os três grupos.

A concentração sérica de proteína total demonstrou diferença aos 29 dias entre os cordeiros submetidos ao manejo de amamentação por 12 horas e os de mamada contínua ( 24 horas). Em relação à concentração sérica de globulinas, notou-se diferença entre os tratamentos no $29^{\circ} \mathrm{e}$ $43^{\circ}$ dias, sendo os valores diferentes entre os cordeiros que permaneciam 24 horas e 12 horas com as mães. As variações encontradas para o proteinograma dos cordeiros Pantaneiros deste trabalho, provavelmente, foram caracterizadas por diminuição da concentração de globulinas.

Com 43 dias de vida, cordeiros que conviviam 12 horas com as ovelhas apresentaram valores de concentração sérica de ureia menores que os animais dos demais tratamentos. De acordo com a evolução etária dos cordeiros, observou-se, nos animais que permaneciam 12 horas com as ovelhas, que a concentração sérica de proteína total apresentou redução entre 29 e 36 dias, com leve aumento no $43^{\circ}$ dia de vida dos animais (Fig. 1-g). A concentração sérica de albumina apresentou diferença para todos os tratamentos de acordo com o crescimento dos cordeiros (Fig. 1a; 1-b; 1-c). Em todos os grupos, houve tendência para redução dos níveis séricos de globulinas, a partir dos 29 dias de vida dos cordeiros (Fig. 1-d; 1-e; 1-f).

Em relação à glicemia, os valores revelaram-se, em todos os tipos de manejo de amamentação, mais elevados aos 15 dias de vida; os menores valores glicêmicos foram observados aos 43 dias (Fig. 2-a; 2-b; 2-c). A concentração sérica de creatinina apresentou elevação de acordo com o avanço da idade dos cordeiros em todos os tratamentos (Fig. 2-d; 2-e; 2-f).

Para o manejo MAM24, a partir dos 36 dias notase evolução nas concentrações séricas de ureia de acordo com o crescimento do cordeiro (Fig. 2-g). A concentração sérica de ureia também elevou-se aos 29 dias de vida no grupo em que os cordeiros permaneciam 12 horas com as mães e apresentou níveis semelhantes nos dias subsequentes (Fig. 2h). A atividade enzimática da AST mostrou-se mais elevada aos 43 dias de vida para o grupo MAM12 (Fig. 2-i). 
a- ALBUMINA MAM24 b- ALBUMINA MAM12 c- ALBUMINA MAM2X30

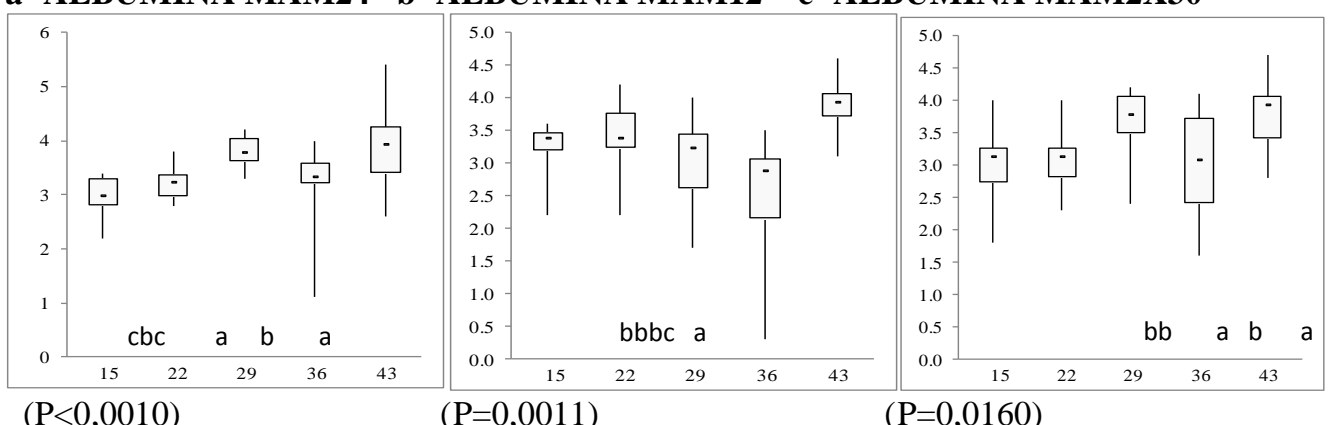

\section{d- GLOBULINAS MAM24e- GLOBULINAS MAM12 f- GLOBULINAS MAM2X30}

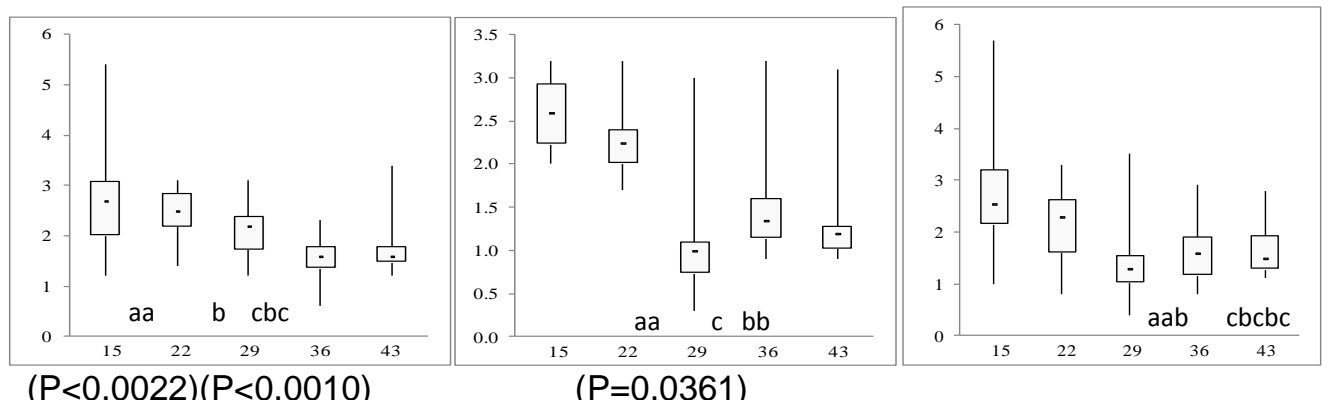

\section{g-PROTEÍNA TOTAL MAM12}

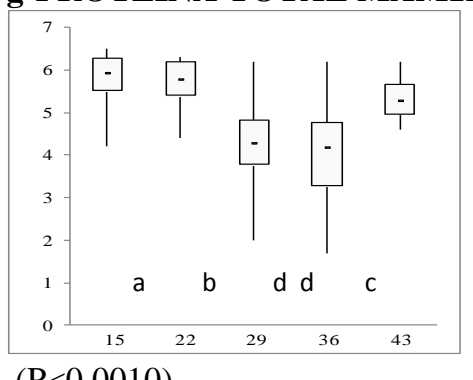

$(\mathrm{P}<0,0010)$

Figura 1. Representação gráfica por box plot das variáveis bioquímicas concentração sérica de albumina, globulinas e proteína total. Para cada variável, letras diferentes indicam diferenças $(\mathrm{P}<0,05)$ entre as idades no teste de Wilcoxon com correção de Bonferroni. Abaixo de cada gráfico, é apresentado o valor-P do teste de Friedman.

\section{DISCUSSÃO}

Para os animais estudados, o manejo nutricional foi determinante para que os cordeiros dos diferentes tratamentos mantivessem ganho de peso semelhante. A suplementação dos cordeiros associada à amamentação demonstrou-se eficiente para o adequado desenvolvimento ponderal dos animais, inclusive os que receberam amamentação controlada. Durante a primeira semana de vida, o cordeiro depende exclusivamente do leite da mãe; após esse período, o animal inicia o consumo de alimentos sólidos, fator que estimula o desenvolvimento ruminal e reduz parcialmente a necessidade de aleitamento (Morales-Terán et al., 2004; Alves et al., 2015). Os diferentes tipos de manejo de amamentação também não influenciaram o peso dos cordeiros em diferentes faixas etárias e o ganho de peso total. 

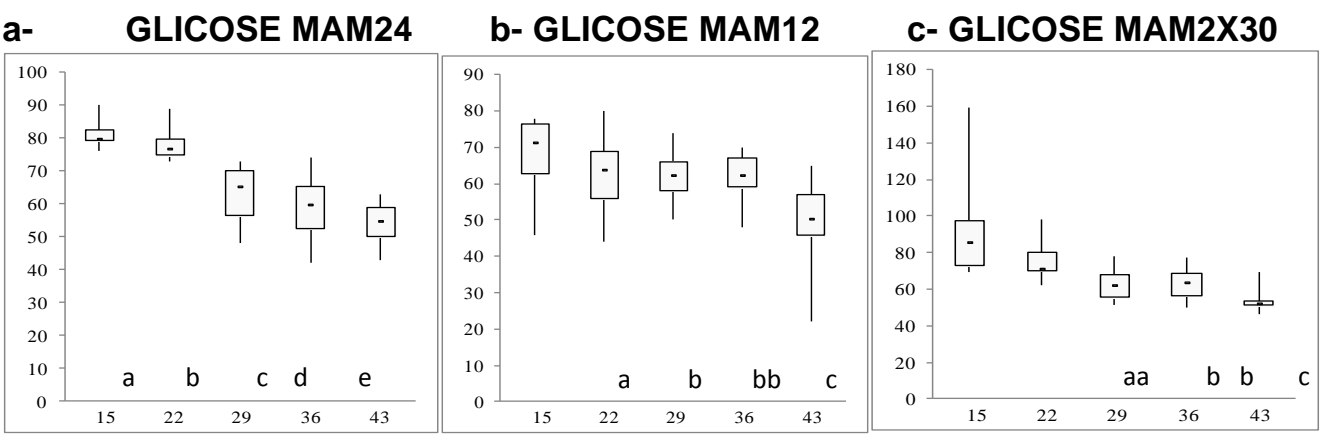

$(P<0,0010) \quad(P=0,0013)$

$(P<0,0010)$

d-CREATININA MAM24 e- CREATININA MAM12 f- CREATININA MAM2X30

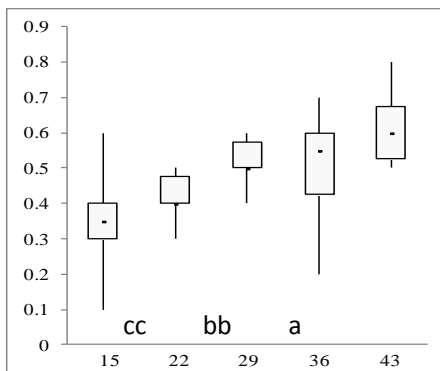

$(\mathrm{P}<0,0010)$

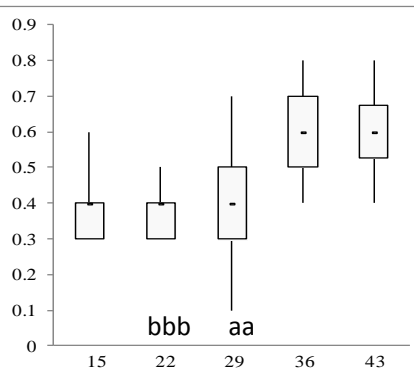

$(\mathrm{P}<0,0010)$

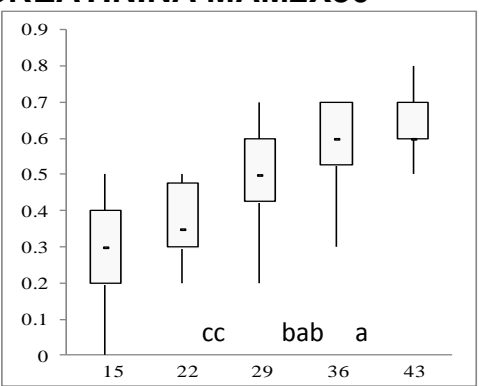

$(\mathrm{P}<0,0010)$

\section{g- UREIA MAM24}

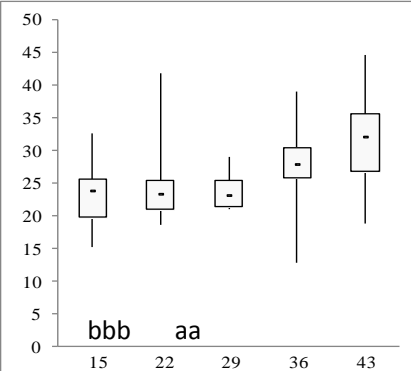

$(\mathrm{P}=0,0081)$

h- UREIA MAM12

i- AST MAM12

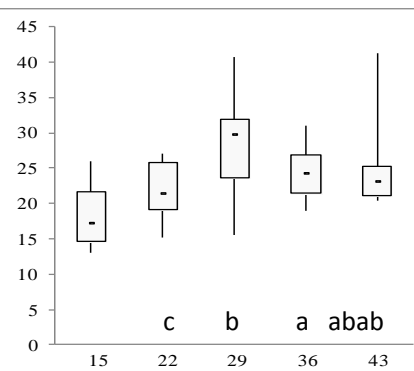

$(\mathrm{P}=0,0055)$

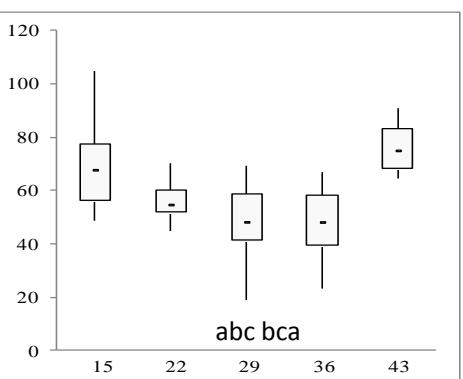

$(P<0,001)$

Figura 2. Representação gráfica por box plot das variáveis bioquímicas glicemia, concentrações séricas de creatinina e ureia e atividade enzimática de aspartato aminotransferase (AST). Para cada variável, letras diferentes indicam diferenças $(\mathrm{P}<0,05)$ entre as idades no teste de Wilcoxon com correção de Bonferroni. Abaixo de cada gráfico, é apresentado o valor-P do teste de Friedman.

A avaliação do eritrograma é importante principalmente para a identificação de quadros de anemia. É de conhecimento notório que em ovinos pode ocorrer o aumento da contagem total de eritrócitos entre 30 e 60 dias de vida (Souza et al., 2018b). As variações entre os valores referentes à contagem de eritrócitos, ao volume globular e à concentração plasmática de hemoglobina acontecem naturalmente ao longo dos dias. Conforme ocorre a lise celular, devido ao envelhecimento natural, novas células são produzidas pela medula óssea (Mohri et al., 2007).

Neste estudo, o aumento da atividade sérica da enzima AST está relacionado à sua presença no colostro e consequente absorção no momento da transferência de imunidade passiva. Em razão de os enterócitos apresentarem a borda em escova, há aumento da capacidade de absorção intestinal nos cordeiros no período pós-natal (Souza et al., 2018a). A variação observada relacionou-se principalmente ao desenvolvimento inicial dos 
processos metabólicos hepáticos para síntese proteica, que são otimizados após o suprimento de colostro. $\mathrm{O}$ amadurecimento fisiológico acontece ao longo das primeiras semanas de vida, enquanto a concentração sérica de globulinas tende a diminuir conforme ocorre a redução da quantidade de anticorpos colostrais (Blum \& Hammon, 2000).

Após a formação da ureia, este metabólito é distribuído e filtrado pelos glomérulos renais e parcialmente reabsorvido pelos túbulos coletores, sendo a principal forma de eliminação de nitrogênio em animais mamíferos (Contreras et al., 2000). Contudo, neste experimento, a variação da concentração sérica de ureia provavelmente ocorreu em função do consumo e da metabolização das proteínas da dieta. A diminuição da concentração sérica de proteína total dos animais com idades entre um e oito meses pode estar relacionada ao decréscimo na concentração das globulinas. E essa redução na concentração sérica de globulinas pode ser inerente ao declínio na concentração de imunoglobulinas fornecidas pela ovelha por meio do colostro (Gonçalves et al., 2001).

A creatinina sérica é produto do metabolismo da musculatura; portanto, os níveis crescentes da concentração sérica de creatinina podem ser explicados pelo desenvolvimento do tecido muscular dos cordeiros com a evolução da idade, visto que não foram observadas alterações clínicas compatíveis com doença renal (Contreras et al., 2000).

\section{CONCLUSÕES}

O acesso dos cordeiros Pantaneiros ao leite materno, por meio de amamentação contínua ou controlada, não interferiu no desempenho ponderal desses animais. Existem variações nos perfis hematológico e bioquímico de cordeiros submetidos a diferentes manejos de amamentação, contudo essas variações não representaram alterações patológicas ou no desenvolvimento de cordeiros Pantaneiros. Foram observadas, porém, variações nos valores hematológicos e bioquímicos de cordeiros Pantaneiros em função do desenvolvimento etário desses animais.

\section{AGRADECIMENTOS}

O presente trabalho foi realizado com apoio da Coordenação de Aperfeiçoamento de Pessoal de Nível Superior - Brasil (Capes) - Código de Financiamento 001.

\section{REFERÊNCIAS}

ALVES, A.C.; ALVES, N.G.; ASCARI, I.J.; JUNQUEIRA, F.B. et al. Colostrum composition of Santa Inês sheep and passive transfer of immunity to lambs. J. Dairy Sci., v.98, p.37063716, 2015.

BLUM, J.W.; HAMMON, H. Colostrum effects on the gastrointestinal tract, and on nutritional, endocrine and metabolic parameters in neonatal calves. Livest. Prod. Sci.,v,66, p.151-159, 2000.

BYERS, S.R.; KRAMER J.W. Normal hematology of sheep and goats . In: WEISS, D.J.; WARDROP, K.J. (Eds.). Schalm's veterinary hematology. Iowa: Blackwell Publishing, 2010. 836p.

CONTRERAS, P.A.; WITTWER, F.; BOHMWALD, H. Uso dos perfis metabólicos no monitoramento nutricional dos ovinos. In: GONZÁLEZ, F.H.D. (Ed.). Perfil metabólico em ruminantes: seu uso em nutrição e doenças nutricionais. Porto Alegre: Universidade Federal do Rio Grande do Sul, 2000. p.75-88.

GONÇALVES, R.C.; PAES, P.R.O.; ALMEIDA, C.T.; FONTEQUE, J.H. et al. Influência da idade e sexo sobre o hemograma, proteínas séricas totais, albumina e globulina de bovinos sadios da raça Guzerá (Bos indicus). Vet. Notícias, v.7, p.61-68, 2001.

LEAL, T.M.; NUNES, J.F.; NASCIMENTO, M.P.S.C.B.; NASCIMENTO, H.T. DOS S. et al. Estro pós-parto em ovelhas da raça Santa Inês. Rev. Cient. Prod. Anim., v,12, p.158-161, 2010.

LONGO, M.L.; VARGAS JUNIOR, F.M.; CANSIAN, K.; SOUZA, M.R. et al. Environmental factors that influence milk production of Pantaneiro ewes and the weight gain of their lambs during the pre-weaning period. Trop. Anim. Health Prod., v.50, p.1250-1254, 2018. 
MOHRI, M.; SHARIFI, K.; EIDI, S. Hematology and serum biochemistry of Holstein dairy calves: age related changes and comparison with blood composition in adults. Res. Vet. Sci., v.83, p.3039, 2007.

MORA, N.H.A.P.; MACEDO, F.D.A.F.; MEXIA, A.A.; ALMEIDA, F.L.A. et al. Características físico-químicas da carne de cordeiras pantaneiras abatidas com diferentes espessuras de gordura subcutânea. Semin. Ciênc. Agrar., v.36, p.2819-2828, 2015.

MORALES-TERÁN, G.; PRO-MARTÍNEZ, A.; FIGUEROA-SANDOVAL, B.; SÁNCHEZDEL-REAL, C. et al. Amamantamiento continuo o restringido y su relación con la duración del anestro postparto en ovejas Pelibuey. Agrociência, v.38, p.165-171, 2004.

OLIVEIRA， D.P.; OLIVEIRA， C.A.L.; MARTINS, E.N. et al. Morphostructural characterization of female and young male of naturalized Sul-mato-grossenses "Pantaneiros" sheep. Semin. Ciênc. Agrar., v.35, p.973-986, 2014.
ŠOCH, M.; BROUČEK, J.; ŠREJBEROVÁ, P. Hematology and blood microelements of sheep in south Bohemia. Biol. Sect. Zool., v.66, p.181-186, 2011.

SOUZA, D.F.; KOWALSKI, L.H.; KULIK, C.H.; BARROS FILHO, I.R. et al. Dinâmicas pré e póscolostral do eritroleucograma, da proteína plasmática total e do fibrinogênio de cordeiros. Ciênc. Anim. Bras., v.19, p.1-11, 2018a.

SOUZA, D.F.; PAULA, E.F.E.; FERNANDES, S.R.; FRANCO, D.R. et al. Dynamics of hematological parameters in female lambs during the first four months of life. Semin. Ciênc. Agrar., v.39, p.2465-2476, 2018b.

VARGAS, F.M.; MARTINS, C.F.; PINTO, G.S.; FERREIRA, M.B. et al. Carcass measurements, non-carcass components and cut production of local Brazilian Pantaneiro sheep and cross breeds of Texel and Santa Inês with Pantaneiro. Small Ruminant Res., v.124, p.55-62, 2015.

ZAR, J.H. Biostatistical analysis. 5.ed. New Jersey: Prentice Hall, 2010. 944p. 